\title{
Evaluation of wall heat flux calculation methods for CFD simulations of an internal combustion engine under both motored and HCCI operation
}

\author{
Gilles Decan $^{\mathrm{a}, *}$, Stijn Broekaert ${ }^{\mathrm{a}}$, Tommaso Lucchini ${ }^{\mathrm{b}}$, Gianluca D’Errico ${ }^{\mathrm{b}}$, Jan Vierendeels ${ }^{\mathrm{a}}$, Sebastian Verhelst ${ }^{\mathrm{a}}$ \\ ${ }^{a}$ Ghent University, Department of Flow, Heat and Combustion Mechanics, Sint-Pietersnieuwstraat 41, 9000 Ghent, Belgium \\ ${ }^{b}$ Politecnico Di Milano, Department of Energy, Via Lambruschini 4, 20156 Milano, Italy
}

\begin{abstract}
In the present work, a study of different numerical heat transfer models is presented used for Homogeneous Charge Compression Ignition (HCCI) internal combustion engine simulations. Since the heat loss through the walls of an engine is an important parameter during engine optimization, as it influences power, efficiency and emissions, accurate modeling techniques need to be available. In this work, the predictive capability of different Computational Fluid Dynamics (CFD) models has been assessed, by using data obtained from experiments on a Cooperative Fuel Research (CFR) engine, a simple single cylinder pancake engine, which has been probed with local heat flux sensors into the combustion chamber walls. The open-source software OpenFOAM ${ }^{\circledR}$ was used to perform simulations of this engine, under both motored and HCCI operation, with a specific focus on the performance of different heat flux models. Due to the simple engine geometry, more numerically demanding heat flux modeling methods could be used, maintaining an acceptable computation time. This allowed a full comparison between the equilibrium wall models as in standard use, an improved empirical heat flux correlation and a numerically intensive low Reynolds formulation. The numerical results considering all aspects of the heat flux - both its progress in time as well as quantitative aspects such as the peak heat flux or the total heat loss - have then been compared to an extensive experimental database. This allowed a full analysis of the performance of the different methods. It was found that the low Reynolds formulation described the physical behavior near the wall the best, while predicting acceptable results concerning the heat flux through the engine walls. The best heat flux prediction was however obtained with an improved empirical model, which additionally has a much shorter computation time. This is crucial when moving on to heat flux simulations of more complex production-type engines. Lastly, the equilibrium models were never capable of accurately predicting the wall heat flux.
\end{abstract}

Keywords: HCCI engine, Computational Fluid Dynamics, Heat transfer, Wall heat flux calculation

\section{Introduction}

Nowadays, the transportation sector is facing prob-

${ }^{*}$ Corresponding author

Email address: gilles.decan@ugent. be (Gilles Decan)

URL: www . ugent. be/users/ gdcan (Gilles Decan)

Preprint submitted to Applied Energy lems due to its negative effects on global warming and human health, caused by the exhaust of excess $\mathrm{CO}_{2}$ 
and harmful emissions like soot and $\mathrm{NO}_{\mathrm{X}}$ into our atmosphere. The bulk of the transport sector is powered by the internal combustion engine. Governing bodies are therefore imposing more stringent emissions legislations, limiting the maximum allowed quantities of $\mathrm{CO}_{2}$ and pollutants an engine can emit. Engine manufacturers have to comply to these rules and are therefore investigating new engine technologies, trying to further develop, improve and optimize them.

An important aspect in the optimization procedure of these engine technologies, such as the HCCI combustion mode, is the study of the heat transfer through the walls of the engine [1, 2, 3, 4, 5]. This has of course a direct influence on the power and efficiency of the engine, since more heat being lost means less power being transferred to the crankshaft. Additionally this also has an influence on the thermal energy that is going out through the exhaust, which is an important aspect since different after-treatment tools require a sufficient activation temperature. Maybe less straightforward is the influence on the formation of pollutants. However, this becomes clear when we see that the formation of for example harmful $\mathrm{NO}_{\mathrm{X}}$ is mostly thermal and thus largely influenced by the temperature in the engine cylinder. It is thus clear that the heat transfer through the walls of an engine is an important aspect in the optimization process and that adequate tools are necessary to characterize and evaluate this.

Computational Fluid Dynamics (CFD) seems like an interesting tool to study this and support the optimization of the engine. It allows a fast change in engine settings and design to determine the optimal ones, without always rebuilding the experimental setup. Of course, the numerical approach has to be validated first, indicating the need for a good initial experimental database.
Other studies, like the one by Komninos et al. [4] and Rakopoulos et al. [6] base themselves on data found in literature, where heat fluxes have been measured during the engine experiments. Such data is for example presented by Nijeweme et al. [2] and Alkidas [7] for spark ignition engines and Lawton [8] for compression ignition engines.

There are a number of problems here. First, only recently have experimental data using more advanced measurements techniques become available for the new combustion modes currently being studied [9]. Thus, modeling approaches for these modes have not yet been validated. Secondly, only the performance of the different heat flux methods under motoring operation was checked previously [4, 6]. The performance of the heat flux models under fired operation, and especially HCCI operation, remains unchecked. There is therefore a need to perform experiments on engines operating according to these new principles and use appropriate measurement tools to obtain reliable and accurate data to evaluate the performance of CFD calculations. Additionally, these simulations need to be performed under both motored but also fired operation, to more accurately evaluate the performance of the heat flux models for a new combustion mode such as HCCI.

Different techniques are currently being used to measure this heat flux. An estimation based on the energy exchange with the cooling circuit [10] can be performed, but this does not give very accurate results. To directly measure the heat flux that is going through the walls of the engine as a function of time, heat flux probes have to be mounted inside the cylinder. These sensors have to be small, since there is not much space in a production engine to insert these probes. A possible solution is the development and use of Thin Film 
Gauge sensors as described by Thorpe et al. [11] and De Cuyper et al. [12, 13]. Their applicability for engine research has been shown by Broekaert et al. [9, 14] by measuring the heat flux in a HCCI operated research engine as well as in a production type engine operating in PPC mode [15]. De Cuyper et al. [16] also demonstrated their use to measure the heat flux in a production type spark ignition engine.

When an extensive database of engine experiments is available, including the heat flux traces, as presented in [14, 9], the performance of different models can be evaluated. First of all, there exist a number of different empirical models, predicting the heat flux in a zerodimensional or one-dimensional manner, like the ones developed by Annand [17], Woschni [18] and Bargende et al. [19]. Where the model of Bargende should be best suited for HCCI operation, it was already shown by Broekaert et al. [14] that all these models are not able to correctly capture the heat flux under varying engine operation. A new model that operates well under varying settings was needed and is described in [15].

Since these empirical models often use simple models for the flow and turbulence in the combustion chamber, improvement to the prediction of the heat flux can also be obtained by switching to three-dimensional models and studying CFD simulations of the internal combustion engine. Also here different models exist to calculate the heat flux through a wall. The earliest developed and probably best known one is the model of Launder and Spalding [20]. This model however uses a lot of simplifications, which is why different others have been developed, for example also taking variable density or viscosity into account. The well-known other heat flux models are the ones of Huh et al. [21], Angelberger et al. [22], Han and Reitz [23] and Rakopoulos et al. [6]. In the work of these last ones, the performance of these CFD models has also been analyzed by comparing them with the gasoline and diesel engine experiments obtained from [2, 7, 8]. They found acceptable results for the models developed by Han and Reitz [23] and Rakopoulos et al. [6], while the results obtained using the other models were inaccurate. Others [10] however state these models overpredict the heat flux at the wall, while Nijeweme et al. [2] and Reitz [24] state the opposite, namely that these models always underpredict the heat flux. It is clear that a thorough investigation coupling experimental and numerical results is necessary.

Additionally, Nijeweme et al. [2] and Ma et al. [25, 26] propose the use of a non-equilibrium approach to calculate the wall heat flux. Contrary to the previously described equilibrium models, no simplifications are used to derive a model. Instead the energy equation is solved in the boundary layer. They compare their results with the ones obtained by using the previously mentioned models and obtained better predictions of the heat flux. Where Ma et al. studied a spark ignition engine, this work wants to focus on an engine using the auto-ignition principle to start its combustion, especially for HCCI operation. In this operation, the flow inside the combustion chamber can be fairly different and can have a large influence on the heat flux through the engine walls. A correct prediction of this heat flux is furthermore very important, as it influences the temperature and thus the start of auto-ignition or combustion, an important control parameter, especially for HCCI operation.

In this work, the different modeling techniques have all been implemented in a CFD framework using OpenFOAM ${ }^{\circledR}$. Results from a motored study of the per- 
formance of these heat flux models have already been presented in [27]. This work then further analyzes the performance of these models under HCCI operation, an interesting combustion mode to tackle global emission problems, however in need of adequate modeling tools for its development and optimization.

In the remainder of this work, the experimental setup together with the used heat flux sensors is first discussed. Secondly, the numerical methodology and a framework and approach for engine simulations is presented. The differently used and most important numerical models for correctly simulating HCCI operation are briefly explained, together with the different heat flux models. Thereafter, the CFD results under varying conditions using various heat flux models are given and compared with experimentally obtained results. The performance and predictive capability of the different models is analyzed and discussed in detail. The findings of this work are then lastly summarized in the conclusion section.

\section{Nomenclature}

Abbreviations

ABDC After Bottom Dead Center

ATDC After Top Dead Center

BBDC Before Bottom Dead Center

BTDC Before Top Dead Center

CAD Crank Angle Degree

CCM Chemistry Coordinate Mapping

CFD Computational Fluid Dynamics

CFR Cooperative Fuel Research
CR Compression Ratio

EGR Exhaust Gas Re-circulation

EVC Exhaust Valve Closing

EVO Exhaust Valve Opening

HCCI Homogeneous Charge Compression Ignition

HFM Hot Film Air Mass

ICE Internal Combustion Engine

IVC Intake Valve Closing

IVO Intake Valve Opening

PIV Particle Image Velocimetry

PPC Partially Premixed Combustion

RANS Reynolds Averaged Navier-Stokes

RNG Re-Normalization Group

RTD Resistance Temperature Detector

SST Shear Stress Transport

TDAC Tabulation of Dynamic Adaptive Chemistry

TFG Thin Film Gauge

TKE Turbulent Kinetic Energy

Symbols

$\alpha$ Thermal diffusivity $\left(m^{2} s^{-1}\right)$

$\alpha_{t}$ Turbulent thermal diffusivity $\left(m^{2} s^{-1}\right)$

$\epsilon$ Eddy-dissipation rate $\left(m^{2} s^{-3}\right)$

$\lambda$ Thermal conductivity $\left(W m^{-1} K^{-1}\right)$ 
$\lambda_{t}$ Turbulent thermal conductivity $\left(W m^{-1} K^{-1}\right)$

$\mu$ Dynamic viscosity (Pas)

$\mu_{t}$ Turbulent dynamic viscosity ( $\left.\mathrm{Pa} s\right)$

$v$ Kinematic viscosity $\left(m^{2} s^{-1}\right)$

$\omega$ Specific dissipation rate $\left(s^{-1}\right)$

$\rho$ Density $\left(\mathrm{kg} \mathrm{m}^{-3}\right)$

$\tau$ Viscous stress tensor $(\mathrm{Pa})$

$\dot{\mathbf{Q}}$ Source term for the rate of heat release $\left(W m^{-3}\right)$

$\mathbf{c}_{\mathbf{p}}$ Specific heat capacity under constant pressure $\left(\mathrm{Jg}^{-1} \mathrm{~K}^{-1}\right)$

$\mathbf{D}_{\mathbf{k}}$ Diffusion coefficient (-)

h Convection coefficient $\left(W m^{-2} K^{-1}\right)$

k Turbulent kinetic energy $\left(m^{2} s^{2}\right)$

Nu Nusselt number (-)

P Pressure $(\mathrm{Pa})$

$\mathbf{P}^{+}$Non-dimensional pressure term (-)

Pr Prandtl number (-)

$\mathbf{P r}_{\mathbf{t}}$ Turbulent Prandtl number (-)

q Heat flux vector $\left(W^{-2}\right)$

R Specific gas constant of air $\left(J \mathrm{~kg}^{-1} \mathrm{~K}^{-1}\right)$

Re Reynolds number (-)

T Temperature $(K)$

$\mathbf{T}_{\mathbf{w}}$ Wall temperature $(K)$
$\mathbf{T}^{+}$Non-dimensional temperature (-)

u Velocity flow field $\left(m s^{-1}\right)$

$\mathbf{u}_{\tau}$ Shear velocity $\left(m s^{-1}\right)$

$\mathbf{u}_{\mathbf{x}}$ Velocity along the $\mathrm{x}$-axis $\left(m \mathrm{~s}^{-1}\right)$

$\mathbf{u}_{\mathbf{y}}$ Velocity along the y-axis $\left(m s^{-1}\right)$

$\mathbf{u}^{+}$Non-dimensional velocity parallel to the wall (-)

y Distance from the wall (m)

$\mathbf{Y}_{\mathbf{k}}$ Species mass fraction (-)

$\mathbf{y}^{+}$Non-dimensional distance from the wall (-)

\section{Experimental setup}

A database of local and instantaneous heat flux measurements has been obtained from a single-cylinder engine, the Waukesha CFR engine, by probing this engine with heat flux sensors. Additionally, also pressure sensors and temperature sensors have been installed in intake and exhaust manifolds as well as inside the cylinder. This resulted in a large database of experimental data, under varying engine conditions, serving as a good tool for comparison and validation of numerical results. This database has been reported in [14]. A short overview of the experimental methodology is first given to better understand how the data was acquired and how it is used for comparison with CFD results.

\subsection{CFR engine}

The CFR engine, which can be seen schematically in Figure 1, is a very simple engine, which makes it perfectly suited for research purposes. It is a flat-piston "pancake combustion chamber" engine, with a single 


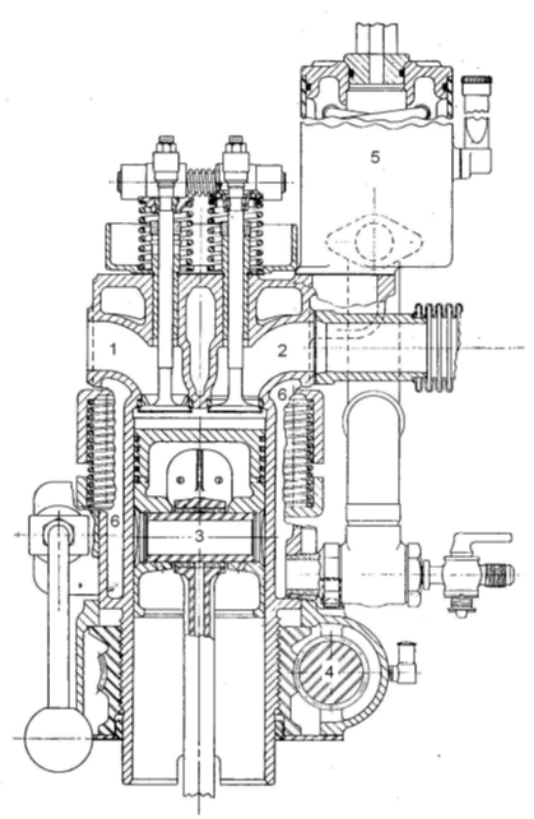

Figure 1: CFR engine

intake and exhaust duct. Its main parameters can be found in Table 1

This engine has been rebuilt for HCCI operation on n-heptane by equipping the intake ducts with a preheating system and an injection system for n-heptane and by removing the throttle valve. Additionally, it also has a variable compression ratio which can be chosen by means of a lever and two values for the engine speed, 600 and $900 \mathrm{rpm}$. For motored operation, this compression ratio and the engine speed have been varied and the heat flux has been recorded accordingly in several probe locations inside the engine cylinder. When the engine was running in HCCI operation, the fuel mass flow rate was also varied. Coupled with measurements of the temperature and pressure, a large experimental database of 12 motored and 22 fired operations under these varying settings was obtained. For the analysis, the measurements, such as the in-cylinder pressure and

\begin{tabular}{|l|l|}
\hline Bore & $83.06 \mathrm{~mm}$ \\
\hline Stroke & $114.2 \mathrm{~mm}$ \\
\hline Connecting rod & $254 \mathrm{~mm}$ \\
\hline Displacement & $618.8 \mathrm{cc}$ \\
\hline Compression ratio & Variable \\
\hline Engine speed & 600 or $900 \mathrm{rpm}$ \\
\hline Intake Valve Opening (IVO) & $10^{\circ}$ ATDC \\
\hline Intake Valve Closure (IVC) & $29^{\circ}$ ABDC \\
\hline Exhaust Valve Opening (EVO) & $39^{\circ}$ BBDC \\
\hline Exhaust Valve Closure (EVC) & $12^{\circ}$ ATDC \\
\hline
\end{tabular}

Table 1: CFR engine parameters

the heat flux, have been recorded for every cycle and are first averaged over 100 cycles. In this work, the graphs always represent this averaged value of the presented quantities. Also, in this work, a base compression ratio of 10 and engine speed of $600 \mathrm{rpm}$ was chosen. If nothing else is mentioned on the operation of the engine, these are the settings that have been used.

\subsection{Heat flux measurements}

The actual and instantaneous heat flux going through the walls of the engine was measured, differently from other works where it is estimated from the heat exchange with the cooling circuit [10]. Two types of sensors have been used for that purpose and have been mounted in the engine cylinder. The first is a commercially available Vatell HFM-7 sensor, which consists of a thermopile to measure the heat flux signal and a Resistance Temperature Detector (RTD). However, due to its rather large dimensions, another type of sensor has been developed, which can be more easily used in a production type engine. This other type of sensor is a Thin Film Gauge sensor, consisting of a thin metallic 
film, which acts as an RTD, on a basis of insulating material [11]. Due to the insulating layer, the heat flux signal cannot be measured directly, but has to be calculated based on the RTD measurements during postprocessing. The interested reader is referred to [12] for more information on this process.

Since the CFR-engine is not a production type engine and space for the mounting of sensors was quite large, the Vatell HFM-7 sensor was mainly used for the heat flux measurements, while the TFGs were used to get an idea of the wall temperatures. The other equipment used during the experiments is described in [14, 9]. An error analysis on the heat flux data was carried out according to the methods described by Taylor [28]. The uncertainty on the measured wall temperature and heat flux is determined by the uncertainties on the calibration coefficients to convert the measured signals to their respective quantities. These coefficients and their uncertainty were determined by the sensor manufacturer. Four possible probe locations have been used, one in the cylinder head (P1), $29.7 \mathrm{~mm}$ off-center, and three in the cylinder liner (P2-P4), centered $9 \mathrm{~mm}$ below the cylinder head. These locations can be seen in Figure 2, where EV and IV represent the exhaust and intake valve locations. If nothing else is mentioned, the heat flux data from sensor location P1 was used.
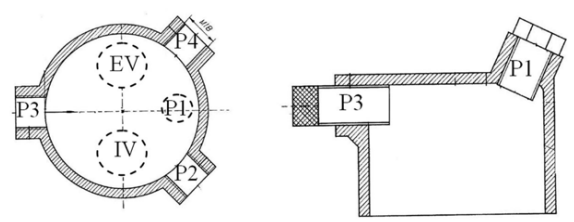

Figure 2: Heat flux sensor locations

\section{Numerical methodology}

Nowadays, 3D-simulations have important tools in engine development and optimization processes. In this work, the OpenFOAM ${ }^{\circledR}$ framework extended with the LibICE library, which incorporates necessary functions for engine simulations and has been developed by the ICE group at Politecnico di Milano [29, 30, 31, 32, 33, 34], has been used. Thermophysical properties such as $c_{p}$, thermal conductivity, thermal diffusivity and viscosity have been modeled in a temperature dependent way and the in OpenFOAM ${ }^{\circledR}$ embedded Sutherland transport model and the JANAF formulation have been used to do so. The ideal gas relation was used to describe the mixture.

Both closed and full cycle engine simulations have been performed, as well as gas exchange simulations. These last ones were necessary to study the effect of possible internal EGR on the species' mass fraction distribution inside the cylinder and investigate any stratification of the fuel. The discretized geometry used for these kind of simulations can be seen in Figure 3 . These gas dynamics simulations served the goal to prop-

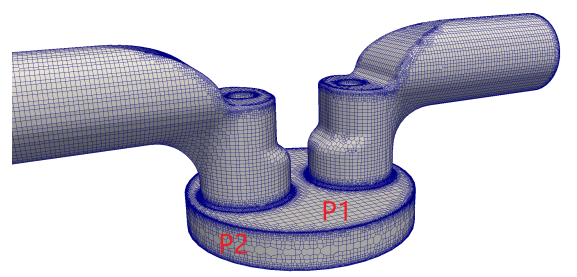

Figure 3: 3D mesh of the CFR engine with an indication of two sensor locations, one in the cylinder head and one in the liner of the engine mesh

erly initialize the species' fields for the afterwards performed closed cycle simulation, where only the internal flow was investigated and the combustion and heat flux 
going through the walls was then studied. Together with the experimental data on the in-cylinder pressure and measured wall temperatures, this allowed to set the initial and boundary conditions for the closed cycle HCCI simulations. In what follows the most important submodels for these simulations will be discussed.

\subsection{Turbulence modeling}

For this work, a RANS methodology was chosen, as it is the least computationally intensive method. This methodology allows the use of a less fine mesh and thus results in a faster computation, ideal for fast engine optimization. This method only resolves the average flow field and requires a model to incorporate the effect of turbulent perturbations. Various models exist, such as the $\mathrm{k}-\epsilon$ model, in its standard, realizable or RNG form, and the k- $\omega$ SST model. For this work, the standard $\mathrm{k}-\epsilon$ model was chosen, with the standard values for its parameters as can be seen in Table 2 Since the main interest was the heat flux modeling and not the turbulence modeling, this model was chosen, because it is the simplest one, performing well in a standard situation. For more information on CFD, the governing equations and how to numerically calculate them and the different turbulence models, the interested reader is referred to specialist literature such as the work of Pope [35], Schlichting [36] or Versteeg and Malalasekera [37].

\subsection{Chemistry modeling}

Transport equations for the chemical species have to be solved in combustion problems taking into account effects of convection, diffusion and reaction. For any chemical species $Y_{k}$, the following equation is solved:

$$
\frac{\partial}{\partial t}\left(\rho Y_{k}\right)+\sum_{i=1}^{3} \frac{\partial}{\partial x_{i}}\left(\rho u_{i} Y_{k}\right)=\sum_{i=1}^{3} \frac{\partial}{\partial x_{i}}\left(\rho D_{k} \frac{\partial Y_{k}}{\partial x_{i}}\right)+\dot{\omega}_{k}
$$

Here the index $i$ represents the three orthonormal directions and $u_{i}$ the velocity in the corresponding direction. The density is given by the term $\rho$, while $D_{k}$ represents the diffusion coefficient, used in Ficks Law to calculate the diffusion flux, and $\dot{\omega}_{k}$ the chemical source term for the species $Y_{k}$ [38]. A chemical kinetic scheme is therefore necessary, to allow the calculation of these source terms. This calculation requires the solution of a set of nonlinear and stiff ordinary differential equations and thus requires a lot of computational resources.

For n-heptane combustion, various kinetic schemes can be used. Largely reduced skeletal mechanisms exist, like the one from Liu et al. [39] consisting of 44 species and 112 reactions, which will result in a fast computation time. However, more detailed kinetic schemes, like the one from Curran et al. [40] consisting of 550 species and 2450 reactions, are capable of predicting more occurring phenomena and thus more accurate results. Since the computation time scales linearly with the number of reactions and quadratically with the number of species [41], this comes however at a very large increase in numerical expenses and is no longer feasible in a straight-forward manner. Some acceleration techniques are necessary if these more detailed kinetic schemes are to be used.

In this work, an extended version of the detailed mechanism of Curran et al. [40] was used, obtained from the website of the Lawrence Livermore National Laboratory and described by Mehl et al. [42, 43]. Since this chemistry scheme contains 652 species and 2822 reactions, chemistry acceleration techniques have to be added to the simulation. A technique based on the TDAC method from Contino et al. [41, 44, 45] has been used. This method stores earlier calculated chemistry solutions in a table and tries to re-use them later 


\begin{tabular}{|l|ccccccc|}
\hline Constant & $C_{\mu}$ & $C_{1}$ & $C_{2}$ & $C_{3}$ & $\sigma_{k}$ & $\sigma_{h}$ & $\sigma_{\epsilon}$ \\
\hline Value & 0.09 & 1.44 & 1.92 & 0 & 1.0 & 1.0 & 0.769 \\
\hline
\end{tabular}

Table 2: Parameter values in k- $\epsilon$ model

on for points in near-equal state. On top of that, a dynamic reduction method is added to the chemistry kinetics scheme, that determines the most important species every time-step and discards the others, thus reducing the computation time when the chemistry does have to be calculated. It has the additional benefit that in this way, the corresponding species equation (1) no longer has to be calculated, further reducing the computation time. Additionally, a Chemistry Coordinate Mapping technique (CCM) as presented in [46, 47] was also used on top of the TDAC method. This allows for multi-zone chemistry, meaning that cells with similar state were grouped and that chemistry only has to be calculated once for the entire group. To not impair the accuracy too much, tabulation was switched off when using this CCM technique.

\subsection{Wall heat flux calculations}

To calculate the heat flux that is going through the walls of the internal combustion engine, the relation given in equation 2 can be used. This is the general way of calculating the heat flux, as a product between the density $\rho$, the specific heat constant $c_{p}$, the thermal diffusivity $\alpha$ and the temperature gradient normal to the wall $d T / d y$.

$$
q_{w}=\rho c_{p} \alpha \frac{d T}{d y_{w}}
$$

However, in a RANS framework, cell sizes are too large to accurately calculate the temperature gradient and sub-grid turbulence modeling is necessary. We can see this adaptation in equation 3 .

$$
q_{w}=\rho c_{p}\left(\alpha+\alpha_{t}\right) \frac{d T}{d y_{w}}
$$

We clearly see the resemblance with the more wellknown relation for the viscous stress $\left(\tau_{w}=\left(\mu+\mu_{t}\right) \frac{d u}{d y}{ }_{w}\right)$, where the viscosity is also divided in a molecular part $\mu$ and a turbulent part $\mu_{t}$, the latter then calculated by the RANS-model. How this turbulent thermal diffusivity $\alpha_{t}$, necessary for heat flux calculations, can be calculated or modeled, will be discussed in the next sections.

\subsubsection{Equilibrium wall models}

The general approach in modeling this turbulent part $\alpha_{t}$ is the use of equilibrium wall models, which can be derived from the thin shear layer energy equation. The obtained equation is then a relation between the temperature and the distance away from the wall, thus describing the behavior of the temperature in the near-wall or boundary layer region. This is the same methodology used to derive the log-law relationship between the velocity and the distance away from the wall in the boundary layer, based on the thin shear layer momentum equation [36]. How these near-wall relations are derived is described by Schlichting [36] and Han and Reitz [23] and is not repeated here. Only the result of these derivations is given, where in equation 4 the well-known momentum law of the wall can be recognized.

$$
u^{+}=\left\{\begin{array}{lll}
y^{+} & \text {if } & y^{+}<11 \\
\frac{1}{\kappa} \ln \left(y^{+}\right)+B & \text { if } & y^{+} \geq 11
\end{array}\right.
$$

This law of the wall gives the relation between a nondimensional velocity $u^{+}$at the wall, which is the ratio 
of the parallel velocity at the wall and the shear velocity $\left(u / u_{\tau}\right)$, and a non-dimensional distance away from the wall $y^{+}\left(y^{+}=y u_{\tau} / v\right)$. It describes the behavior of the velocity in the boundary layer. For more in-depth information on this boundary layer modeling, the interested reader is referred to the work of Schlichting [36].

The solution of the thin shear layer energy equation is less uniform, since a number of different models have been proposed that use different simplifications. The derivation process, described in [23], is however similar for all models and is omitted here. The solution presented here is the model given by Rakopoulos et al. [6], which results in the description of the temperature boundary layer given by equation 5 .

$$
\begin{gathered}
T^{+}=\frac{1}{0.4767}\left[\ln \left(y^{+}+\frac{1}{0.4767 P r}\right)-\ln \left(40+\frac{1}{0.4767 P r}\right)\right] \\
+10.2384+P^{+}\left(\frac{y^{+}-40+117.31\left(0.4767+\frac{1}{P r}\right)}{0.4767+\frac{1}{P r}}\right)
\end{gathered}
$$

Again, a relation between a non-dimensional quantity, this time the temperature $T^{+}$and a non-dimensional distance $y^{+}$is obtained. One can also recognize the first part of the equation presenting a logarithmic relation while the second part is a linear relation, indicating a similar behaviour of the temperature in the boundary layer as prescribed by the momentum law of the wall in equation 4

By using the relation between $T^{+}$and the heat flux and the definition of $P^{+}$, which represent the influence of pressure fluctuations $\left(\frac{d P}{d t}\right)$ as given by equation 6 , a formulation for the wall heat flux (7) can be derived from this temperature boundary layer profile [6].

$$
T^{+}=\frac{\rho u_{\tau} c_{p} T}{q_{w}} \ln \left(\frac{T_{w}}{T}\right) ; \quad P^{+}=\frac{\left(\frac{d P}{d t}\right) v}{q_{w} u_{\tau}}
$$

$q_{w}=\frac{\rho c_{p} u_{\tau} T \ln \left(\frac{T_{w}}{T}\right)-\frac{d P}{d t} \frac{v}{u_{\tau}}\left(\frac{y^{+}-40}{0.4767+\frac{1}{P r}}+117.31\right)}{\frac{1}{0.4767}\left[\ln \left(y^{+}+\frac{1}{0.4767 P r}\right)-\ln \left(40+\frac{1}{0.4767 P r}\right)\right]+10.2384}$

By definition, equation 7 should be equal to equation 3. which allows the calculation of the turbulent thermal diffusivity $\alpha_{t}$ at the wall and thus allows the modeling of the turbulent contribution to the heat flux.

Besides the wall heat flux model described by Rakopoulos et al. [6], other models exist, like the ones from Angelberger et al. [22], Han and Reitz [23], Huh et al. [21] and Launder and Spalding [20]. The formulation of these wall models is presented in Appendix A. The performance of these models have all been analyzed and is presented later on.

These wall models have also been compared to a much simpler method, where a straightforward value for the turbulent thermal diffusivity $\alpha_{t}$ is set. This value is given as the ratio of the turbulent viscosity $\mu_{t}$, which is modeled by the turbulence model, to the turbulent Prandtl number $P r_{t}$ which was chosen constant. This method omitted the use of these complex wall heat flux formulations and allowed the choice of the most appropriate turbulent Prandtl number to best match the experimentally obtained heat flux results.

\subsubsection{Convective heat flux modeling}

Another approach in the calculation of the heat flux, is the use of empirical correlations. These are related to the convection coefficient and therefore calculate the heat flux based on the convective law given in equation 8.

$$
q_{w}=h \cdot\left(T_{g a s}-T_{w}\right)
$$

The convection coefficient $h$ in this equation can be found by using the Pohlhausen equation, relating the 
Nusselt number to the Reynolds and Prandtl number 99.

$$
\frac{h L}{\lambda}=N u=a \operatorname{Re}^{b} \operatorname{Pr}^{c}
$$

Various empirical models exist, such as the ones from Annand [17], Woschni [18] or Bargende [19], where values for the parameter $a, b$ and $c$ are chosen. Here $c$ is mostly chosen 0 , since the Prandtl number does not change significantly during the engine cycle [48] and can thus be lumped into the parameter $a$. Additionally, since $R e=\rho U L / \mu$, the characteristic length $L$ and velocity $U$ have to be modeled to accurately represent the gas motion inside the engine.

In this work, a convective heat flux formulation has also been implemented in OpenFOAM ${ }^{\circledR}$. The chosen model for the derivation of the convection coefficient is the one by Broekaert et al. [15]. Here the characteristic length was chosen equal to the bore of the engine, while the characteristic velocity was modeled in time, equal to the square root of twice the average turbulent kinetic energy in the engine cylinder $(U=\sqrt{2 k})$. The model for the convection coefficient then looks like:

$$
h=a \frac{\lambda}{D}\left(\frac{\rho \sqrt{2 k} D}{\mu}\right)^{b}
$$

with $D$ being the engine bore, $a$ being an engine dependent scaling coefficient, chosen equal to 0.15 for the CFR engine and $b$ a constant chosen equal to 0.8 . It was empirically shown in [15, 14] that this model performed the best for new combustion modes such as HCCI and PPC, and it is therefore also compared in this work in a CFD framework.

\subsubsection{Low Reynolds approach}

A final method that has been studied, is to no longer use any model or correlation, but to refine the mesh in the boundary layer region and thus accurately resolve the temperature in that region and therefore also the temperature gradient. This is achievable when reducing the $y^{+}$value below 5 , to resolve the thermo-viscous sublayer.

With this method, it is no longer necessary to model the turbulent thermal diffusivity $\alpha_{t}$, since the cell sizes are refined and the temperature gradient is now accurately calculated by the simulation. Results on this method and the other previously described methods will be presented in the next section. An important note however already on this Low Reynolds approach, is that the large increase in mesh resolution makes this method very numerically demanding, which is something that has to be taken into account.

\section{Results and discussion}

\subsection{Validation}

Before comparing and analyzing the different heat flux calculation methods, it is necessary to ensure that the proper operation of the HCCI engine has been simulated. Obtained numerical results on important engine quantities have to be validated with the corresponding experimental ones, for a case where the CFR engine was operating at $600 \mathrm{rpm}$ with a compression ratio of 10 .

The first and most important quantity that can be compared, is the value for the in-cylinder pressure throughout the engine cycle, which has been measured experimentally with a measurement error of $1 \%$. An average pressure trace can then be displayed together with the obtained traces from the performed simulations, which can be seen in Figure 4 In this figure, the pressure obtained using different methodologies to calculate the heat flux have all been plotted, where only the pressure trace from one equilibrium wall model has 


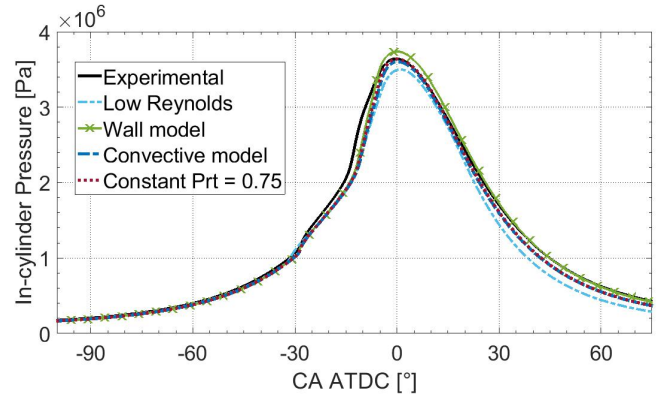

Figure 4: Pressure trace under HCCI operation with $\mathrm{CR}=10$ and running at $600 \mathrm{rpm}$

been plotted to prevent cluttering the figure. It was however found that all simulations with different wall models gave the same pressure trace. Furthermore, it is clear that the different methods all predict the pressure in an accurate manner as the corresponding pressure traces nearly overlap with the experimental one. This is already a good indication that the correct engine operation has been simulated. For motored simulations, this comparison of the pressure trace is the only validation tool at hand, indicating the importance of a good correspondence between numerical and experimental pressure traces. This validation has also been done for motored operation, where a near-perfect overlap between numerical and experimental results was found.

A second quantity that can be compared, but only during combustion simulations, is the rate of heat release inside the cylinder, which has been plotted in Figure 5 It allows the validation of the combustion timing. Here the experimental trace is again compared to the different numerical ones, where again only the results from one simulation with an equilibrium wall model have been displayed. In Figure 5 it is clear that the two important instances where combustion takes place (twostep heat release) are well predicted by all but one simulation, where only the first peak with the low Reynolds

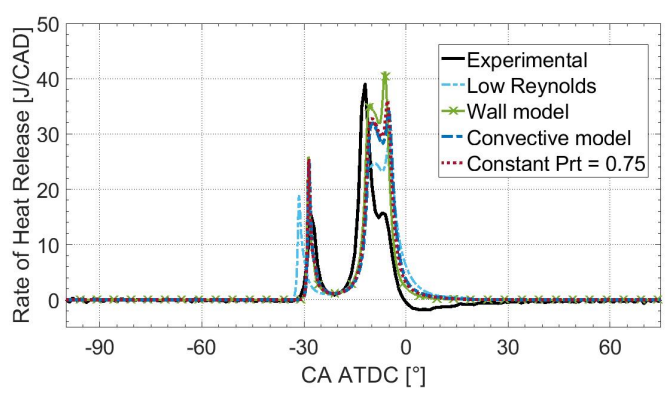

Figure 5: Rate of heat release under HCCI operation with $\mathrm{CR}=10$ and running at $600 \mathrm{rpm}$

method is slightly early. Furthermore, also the peak values of the heat release rate are well predicted by all models.

There is however a difference in total amount of heat being released between the simulations and the experiment. The simulations all represent the gross rate of heat release rate, calculated from the mass of fuel being burned and the lower heating value of n-heptane. The experimental heat release rate however represents the net rate of heat release, taking heat losses into account. This resulted in a total cumulative heat release of $372 \mathrm{~J}$ and $240 \mathrm{~J}$ respectively. These values seem to be right since the total energy content of the injected fuel is 376 $\mathrm{J}$, which differs from the found $372 \mathrm{~J}$ due to combustion inefficiencies. The difference of $132 \mathrm{~J}$ between the simulated and experimental cumulative heat release is then the total heat loss. This value is confirmed later on in this paper.

One can note that these heat losses are quite high and represent a loss of $35 \%$. This is due to the combustion occurring mainly before TDC, as can be seen in Figure 5. which causes the high efficiency loss. The focus of this work was however not on optimizing the combustion and its phasing, but to perform a study of the heat flux and how to model it. This will allow a correct mod- 
eling of HCCI operation to develop and optimize such an engine in the future.

Lastly, also the general methodology used for the simulation of internal combustion engines has to be validated. This has been conducted by performing some simulations of other engine geometries, such as the ones described in [7, 8], and comparing the results to other published results. This validation aspect has already been reported in a previous work [27]. Furthermore, this previous work also performed a mesh dependency check for the low Reynolds methodology, resulting in an appropriate mesh, which has been used here as well. The cell count for this low Reynolds mesh, with fine layering at the walls, was 40,000 for a $5^{\circ}$ sector mesh at TDC, 20 times more than the used mesh for the other methods.

Since the pressure traces overlap well, and the combustion timing is well predicted, letting aside the small early ignition found with the Low Reynolds model, it was concluded that a correct operation of the CFR engine has been simulated in all cases. Heat flux results can therefore be objectively compared and analyzed, which is presented in the next section.

\subsection{Heat flux results}

Due to the inserts of local heat flux probes inside the engine cylinder, the local and instantaneous heat flux going through the walls of the engine can be analyzed. The heat flux can then be given as a function of crank angle, where $0^{\circ}$ represents top dead center. This has been done to study HCCI operation and analyze the performance of current heat flux modeling tools for this new combustion mode. The heat flux curves can be seen in Figure 6. The results obtained from the experiments are given in the black solid lines in this figure, where also the error bars, representing the measurement inaccuracy from the heat flux sensor as described in Section 2.2. have been indicated.

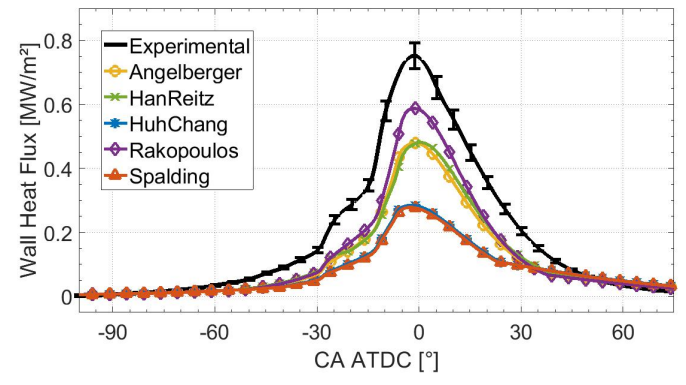

(a) Equilibrium wall models

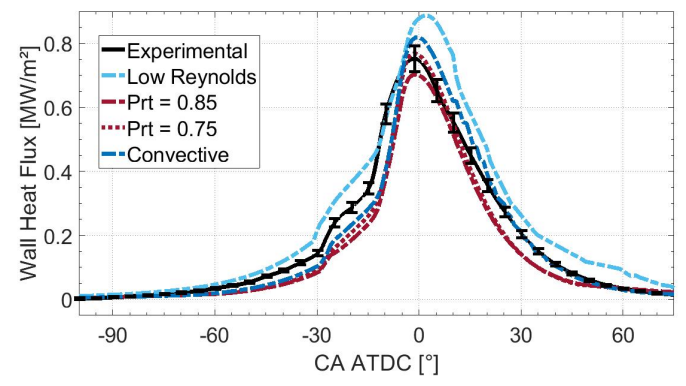

(b) Constant $\operatorname{Pr}_{t} /$ Convective / Low Reynolds

Figure 6: Wall heat flux results under HCCI operation with $\mathrm{CR}=10$ and running at $600 \mathrm{rpm}$ for (a) the equilibrium wall models and (b) turbulent Prandtl, convective and low Reynolds modeling.

When investigating the performance of the wall models in Figure 6a, one can see the inability to accurately predict the heat flux throughout the engine cycle. In all stages of the closed cycle, from compression over combustion around top dead center to the expansion phase, these models underpredict the heat flux that goes through the walls of the engine. The models from Han and Reitz [23] and Rakopoulos et al. [6] perform the best, as was expected, but their results are still not representative of the actual wall heat flux.

As was stated before, results were also obtained by no longer using a wall model to model the turbulent 
thermal diffusivity $\alpha_{t}$, but by using a constant turbulent Prandtl number and to simply calculate $\alpha_{t}$ as the ratio of the turbulent viscosity $\mu_{t}$ over $\operatorname{Pr}_{t}$. This also allowed some tuning of the results by adjusting the used value of $\operatorname{Pr}_{t}$, something which was not possible with the equilibrium wall models. Results are presented in Figure 6b, where the turbulent Prandtl number has been changed from 0.85 to 0.75 . A similar behavior compared to the wall models is obtained, where both during compression and expansion results are underpredicted. However, due to the tweaking of the turbulent Prandtl number, by adjusting it from 0.85 to 0.75 , the peak heat flux around top dead center can be better predicted. It is then however important that this value of $P r_{t}$ is the optimal one in a range of operation conditions and that its use can be extended from this one case for which it was tuned. Figures 7 and 8 therefore present the total heat loss through the walls, obtained by multiplying the heat flux results with the instantaneous cylinder surface area and integrating those over time, and the peak heat flux for varying compression ratio and engine speed. From these figures, it is clear that the success of this method is arbitrary, where good results considering the heat loss or the peak heat flux are achieved in certain situations, but that this is certainly not extendable to the whole operation range.

When using an empirical relation based on the convective law as developed by Broekaert [15], better results are obtained. While it is clear from Figure $6 \mathrm{~b}$ that the numerical results still underpredict the heat flux during the compression phase, a good prediction is found from around top dead center until the end of the expansion stroke. Furthermore, the model also performs well when investigating it under varying compression ratio and engine speed, as can be seen in Figures 7 and 8 Val- ues more or less within the error margin are predicted. Additionally, also the same trends as those found during experiments are found using this method. For example, while other methods predict a larger increase in total heat loss when increasing the compression ratio from 10 to 11 than from 9 to 10 , the opposite is found with the convective modeling approach. This is also the trend that is experimentally found, as can be seen in Figure $7 \mathrm{a}$. This leads us to the conclusion that this model performs well and is best suited for a fast investigation of the heat transfer in an engine.

A last method was however also investigated, which resolves the near-wall behavior, reducing the need for the modeling of the heat flux since the temperature gradient is more accurately calculated. This however drastically increased the computation time. While the other methods all had a computation time of about 80 processor-hours for the simulation of the closed cycle of the HCCI engine, using a RANS methodology and axi-symmetry, the low Reynolds approach had a computation time of about 1000 processor-hours. This already indicates the large increase in computationally required resources, which was still manageable for axisymmetric RANS simulations, but is no longer suited for complete engine simulations. The heat flux through the walls of the engine is slightly better predicted, as is shown in Figure 6b. Throughout the engine cycle, heat flux results are representative of what was found during experiments and the trace more distinctively represents different engine phases. Especially during the compression phase, where other methods always seem to underpredict the heat flux and do not capture the first heat flux increase, this method seems to capture the experimental trends. A slight overprediction is however obtained when comparing the numerical results to the experimen- 


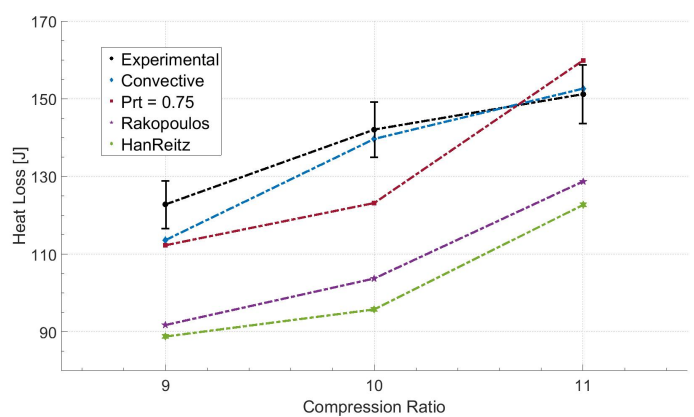

(a) 600 RPM

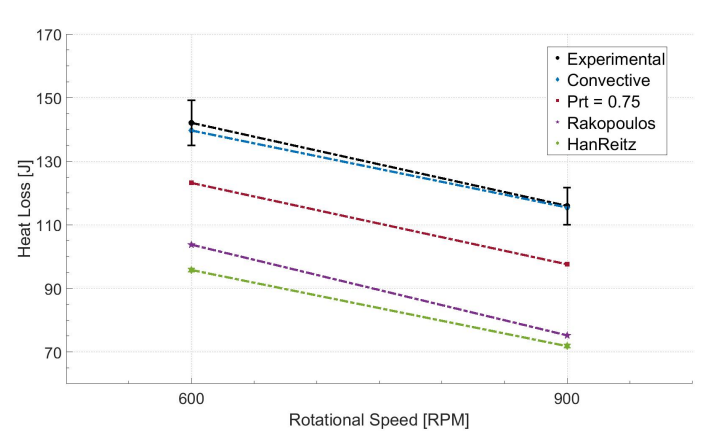

(b) $\mathrm{CR}=10$

Figure 7: Heat loss through the engine walls for different models, in one HCCI cycle under varying engine conditions. (a) under varying compression ratio for fixed rotational speed, (b) under varying rotational speed and fixed compression ratio.

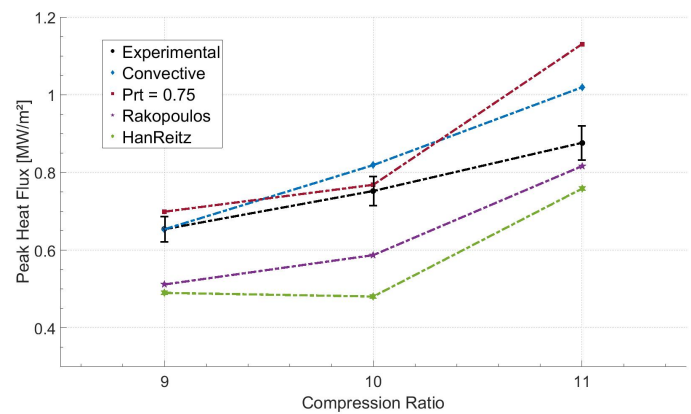

(a) 600 RPM

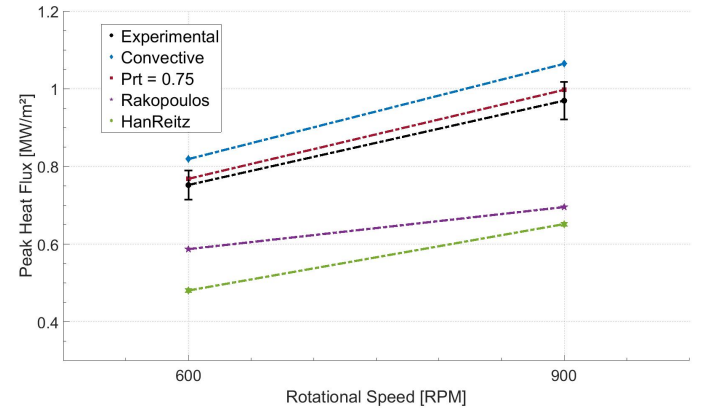

(b) $\mathrm{CR}=10$

Figure 8: Peak heat flux through the walls of the HCCI engine, obtained with different models under varying engine conditions. (a) under varying compression ratio for fixed rotational speed, (b) under varying rotational speed and fixed compression ratio.

tal ones. This method also gave the most accurate heat flux predictions under motored operation, as was reported in a previous work [27]. If a study of the heat transfer is to be performed and RANS simulations using axi-symmetry can be performed, the computational time is still acceptable and this method can still be considered.

\subsection{Temperature profiles}

To further analyze the performance of the different heat flux calculation methods, the behavior of the temperature in the near-wall region has been studied. How the temperature behaves can be seen in Figure 9, for three different instances, one during compression at $25^{\circ} \mathrm{CAD}$, one at TDC and one during expansion at $15^{\circ}$ CAD.

Even though the wall models prescribe a linear and logarithmic relation between the temperature and the distance away from the wall in equation 5 and Table A.1. it is clear that during the simulation this is not retrieved. Due to an insufficient mesh resolution, this is not possible and an incorrect temperature gradient is found. This was however expected and a turbulent thermal diffusivity was modeled to counteract this. It is thus 


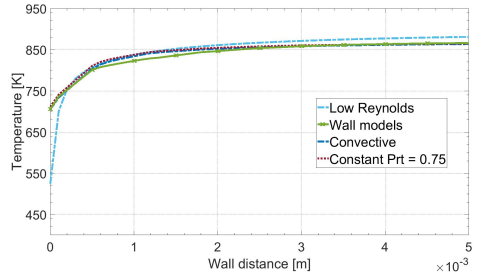

(a) $\mathrm{CAD}=-25^{\circ}$

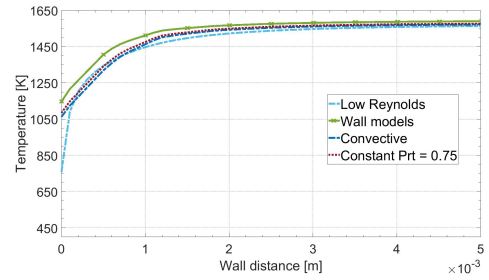

(b) $\mathrm{CAD}=0^{\circ}$

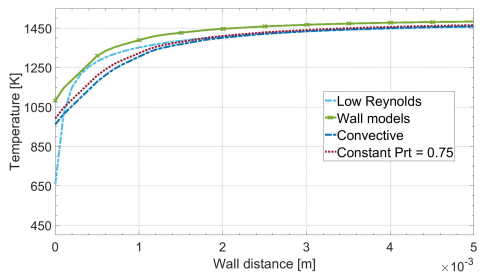

(c) $\mathrm{CAD}=15^{\circ}$

Figure 9: Boundary layer temperature profiles for different heat flux calculation methods at different instances under HCCI operation with CR = 10 and running at $600 \mathrm{rpm}$

clear that this $\alpha_{t}$ is not capable of counteracting this incorrect gradient and that the heat flux prediction is inaccurate.

Lower temperatures are obtained with the convective modeling approach, especially at $15^{\circ} \mathrm{CAD}$, which is during the expansion phase. Lower temperatures are obtained both at the wall and in the bulk, indicating an increased heat flux. Compared to the wall model, the difference is larger at the wall than in the bulk, which therefore results in a larger temperature difference between the wall and the bulk. This explains the better prediction of the heat flux. It is however only the low Reynolds approach that can accurately represent the temperature behavior in the boundary layer region, due to the increased mesh resolution. This difference is largest at $-25^{\circ} \mathrm{CAD}$, which explains the heat flux effects that are captured during compression by this method, as was previously explained and shown in Figure 6 Furthermore, also the velocity and turbulent kinetic energy profiles resemble the expected linear-logarithmic relation, with as result the prediction of a smaller value at the wall but a larger value for the bulk. These profiles are however omitted here, as they look similar to the ones in Figure 9. As the velocity difference and this turbulent kinetic energy are a measure for the convective part of the heat flux, bigger values result in a larger heat flux. While most accurately representing the physical behavior at the walls in the engine, this method however resulted in a slightly too large heat flux, as was already seen before.

\section{Summary and conclusions}

In the present work, a study of different numerical heat transfer models is presented used for internal combustion engine simulations. Simulations of a Cooperative Fuel Research engine have been carried out using these models, when the engine was operated under both motored and Homogeneous Charge Compression Ignition operation. Due to the existence of a large experimental database, consisting of local and instantaneous heat flux measurements, a performance analysis of the different calculation methods could be carried out.

It was found that the equilibrium wall models were never able of correctly predicting the heat flux through the walls of the HCCI engine. The temperature gradient was not accurately resolved, as was expected, but the turbulent modeling near the wall was not able of counter-acting this. Due to the simplifications used in deriving the wall heat flux models, some occurring effects can no longer be captured. Furthermore, it was found that by replacing the turbulent modeling by the ra- 
tio of the turbulent viscosity and Prandtl number, comparable and even slightly better results are achieved.

Good results have been obtained by using a newly developed empirical correlation as well as with a low Reynolds approach. However, when performing a full engine Computational Fluid Dynamics optimization study, the low Reynolds approach is not a viable option due to the large computational cost. It is the authors' opinion that, when developing an HCCI engine to reduce emissions and investigating the heat losses, the presented convective model should be used. When however an optimization of the heat flux is solely performed and axi-symmetry can be used, a low Reynolds approach can be affordable and is advised, to accurately describe the physical behavior at the wall. Combined with the convective modeling approach, this gives the best prediction of the heat flux through the walls of an Homogeneous Charge Compression Ignition engine.

\section{Acknowledgements}

This research has been funded by Ghent University (Belgium) through GOA project [BOF16/GOA/004]. The authors gratefully acknowledge the financial support.

\section{References}

[1] J. Dec, Advanced compression-ignition engines - understanding the in-cylinder processes, Pro Combustion Inst 32 (2009) 27272742. doi:10.1016/j.proci.2008.08.008

[2] D. O. Nijeweme, J. Kok, C. Stone, L. Wyszynski, Unsteady incylinder heat transfer in a spark ignition engine: experiments and modelling, Proc Inst Mech Eng, Part D 215 (6) (2001) 747760. doi:10.1243/0954407011528329

[3] G. Borman, K. Nishiwaki, Internal-combustion engine heattransfer, Prog Energy Combustion Sci 11 (4) (1987) 1-46. doi: $0.1016 / 0360-1285(87) 90005-0$
[4] N. Komninos, G. Kosmadakis, Heat transfer in hcci multi-zone modeling: Validation of a new wall heat flux correlation under motoring conditions, Appl Energy 88 (5) (2011) 1635-1648. doi:10.1016/j.apenergy.2010.11.039

[5] N. Komninos, C. Rakopoulos, Heat transfer in hcci phenomenological simulation models: A review, Appl Energy 181 (2016) 179-209. doi:10.1016/j . apenergy . 2016.08.061

[6] C. Rakopoulos, G. Kosmadakis, E. Pariotis, Critical evaluation of current heat transfer models used in cfd in-cylinder engine simulations and establishment of a comprehensive wall-function formulation, Appl Energy 87 (5) (2010) 1612-1630. doi:10. 1016/j . apenergy . 2009.09.029

[7] A. Alkidas, Heat transfer characteristics of a spark-ignition engine, J Heat Transfer 102 (2) (1980) 189-193. doi:10.1115/ 1.3244258

[8] B. Lawton, Effect of compression and expansion on instantaneous heat transfer in reciprocating internal combustion engines, Proc Inst Mech Eng, Part A 201 (3) (1987) 175-186. doi:10.1243/PIME_PROC_1987_201_022_02

[9] S. Broekaert, T. D. Cuyper, M. D. Paepe, S. Verhelst, Experimental investigation of the effect of engine settings on the wall heat flux during heci combustion, Energy 116 (1) (2016) 10771086. doi:10.1016/j.energy.2016.10.042

[10] F. Berni, G. Cicalese, S. Fontanesi, A fully coupled computational fluid dynamics and multi-zone model with detailed chemical kinetics for the simulation of premixed charge compression ignition engines, Appl Therm Eng 115 (2017) 1045-1062. doi:10.1016/j.applthermaleng.2017.01.055

[11] S. Thorpe, S. Yoshino, R. Ainsworth, N. Harvey, Improved fast-response heat transfer instrumentation for short-duration wind tunnels, Meas Sci Technol 15 (2004) 1897-1909. doi: 10.1088/0957-0233/15/9/030

[12] T. D. Cuyper, S. Bracke, J. Lavens, S. Broekaert, K. Chana, M. D. Paepe, S. Verhelst, Demonstrating the use of thin film gauges for heat flux measurements in ices: Measurements on an inlet valve in motored operation, SAE Technical Paper 2016-010641 (2016) 1- doi:10.4271/2016-01-0641

[13] T. D. Cuyper, G. Fossaert, O. Collet, S. Broekaert, K. Chana, M. D. Paepe, S. Verhelst, Calibration of a tfg sensor for heat flux measurements in a s.i. engine, SAE Technical Paper 201501-1645 (2015) 1-Cdoi:10.4271/2015-01-1645

[14] S. Broekaert, T. D. Cuyper, M. D. Paepe, S. Verhelst, Evaluation of empirical heat transfer models for hcci combustion in a $\mathrm{cfr}$ 
engine, Appl Energy 205 (2017) 1141-1150. doi:10.1016/ j.apenergy.2017.08.100

[15] S. Broekaert, M. D. Paepe, S. Verhelst, A heat transfer model for low temperature combustion engines, SAE Technical Paper 2018-01-1662 (2018) 1-13doi:10.4271/2018-01-1662.

[16] T. D. Cuyper, S. Broekaert, K. Chana, M. D. Paepe, S. Verhelst, Evaluation of empirical heat transfer models using tfg heat flux sensors, Appl Therm Eng 118 (2017) 561-569. doi:10.1016/ j.applthermaleng.2017.02.049

[17] W. Annand, Heat transfer in the cylinders of reciprocating internal combustion engines, Proc Inst Mech Eng 177 (1) (1963) 973-996. doi:10.1243/PIME_PROC_1963_177_069_02

[18] G. Woschni, Universally applicable equation for the instantaneous heat transfer coefficient in the internal combustion engine, SAE Technical Paper 670931doi:10.4271/670931

[19] M. Heinle, M. Bargende, H. Berner, Some useful additions to calculate the wall heat losses in real cycle simulations, SAE Int J Engines 5 (2) (2012) 469-482. doi: 10.4271/2012-01-0673

[20] B. Launder, D. Spalding, The numerical computation of turbulent flows, Comput Methods Appl Mech Eng 3 (2) (1974) 269289. doi:10.1016/0045-7825(74)90029-2

[21] K. Huh, I. Chang, J. Martin, A comparison of boundary layer treatments for heat transfer in ic engines, SAE Technical Paper 900252doi:10.1016/j.apenergy.2009.09.029

[22] C. Angelberger, T. Poinsot, B. Delhay, Improving near-wall combustion and wall heat transfer modeling in si engine computations, SAE Technical Paper 972881doi:10.4271/900252

[23] Z. Han, R. Reitz, A temperature wall function formulation for variable-density turbulent flows with application to engine convective heat transfer modeling, Inl J Heat Mass Transfer 40 (3) (1997) 613-625. doi:10.1016/0017-9310(96)00117-2

[24] R. Reitz, Assessment of wall heat transfer models for premixedcharge engine combustion computations, SAE Technical Paper 91026 doi:10.4271/910267

[25] P. Ma, T. Ewan, C. Jainski, L. Lu, A. Dreizler, V. Sick, M. Ihme, Development and analysis of wall models for internal combustion engine simulations using high-speed micro-piv measurements, Flow, Turbulence and Combustion 98 (1) (2017) 283309. doi:10.1007/s10494-016-9734-5

[26] P. Ma, M. Greene, V. Sick, M. Ihme, Non-equilibrium wallmodeling for internal combustion engine simulations with wall heat transfer, Int J Engine Res (2017) 1-11doi:10.1177/ 1468087416686699
[27] G. Decan, S. Broekaert, T. Lucchini, G. D’Errico, J. Vierendeels, S. Verhelst, Evaluation of wall heat flux models for full cycle cfd simulation of internal combustion engines under motoring operation, SAE Technical Paper 2017-24-0032 (2017) 112 doi: 10.4271/2017-24-0032

[28] J. Taylor, An Introduction to Error Analysis: The Study of Uncertainties in Physical Measurements, 2nd Edition, University Science Books, 1997, ISBN: 9780935702750.

[29] T. Lucchini, G. D'Errico, D. Ettorre, Numerical investigation of the spraymeshturbulence interactions for high-pressure, evaporating sprays at engine conditions, Int J Heat Fluid Flow 32 (2011) 285-297.doi:10.1016/j.ijheatfluidflow. 2010. 07.006

[30] H. Ismail, H. Ng, S. Gan, T. Lucchini, Computational study of biodieseldiesel fuel blends on emission characteristics for a light-duty diesel engine using openfoam, Appl Energy 111 (2013) 827-841. doi:10.1016/j . apenergy . 2013.05.068

[31] L. Cornolti, A. Onorati, T. Cerri, G. Montenegro, F. Piscaglia, $1 \mathrm{~d}$ simulation of a turbocharged diesel engine with comparison of short and long egr route solutions, Appl Energy 111 (2013) 1-15. doi:10.1016/j.apenergy . 2013.04.016

[32] T. Lucchini, G. D'Errico, D. Ettorre, F. Brusiani, G. Bianchi, A. Montanaro, L. Allocca, Experimental and numerical investigation of high-pressure diesel sprays with multiple injections at engine conditions, SAE Technical Paper 2010-01-017, doi: 10.4271/2010-01-0179

[33] T. Lucchini, G. D’Errico, M. Fiocco, Multi-dimensional modeling of gas exchange and fuel-air mixing processes in a directinjection, gas fueled engine, SAE Technical Paper 2011-240036 doi:10.4271/2011-24-0036

[34] T. Lucchini, G. D'Errico, T. Cerri, A. Onorati, G. Hardy, Experimental validation of combustion models for diesel engines based on tabulated kinetics in a wide range of operating conditions, SAE Technical Paper 2017-24-002 doi : 10.4271/ 2017-24-0029

[35] S. Pope, Turbulent Flows, 1st Edition, Cambridge University Press, 2000, ISBN: 9780521598866.

[36] H. Schlichting, K. Gersten, Boundary-layer theory, 8th Edition, Springer, 2000, ISBN: 9783540662709.

[37] H. Versteeg, W. Malalasekera, An Introduction to Computational Fluid Dynamics: The Finite Volume Method, 2nd Edition, Pearson, 2007, ISBN: 9780131274983.

[38] T. Poinsot, D. Veynante, Theoretical and Numerical Combus- 
[39] S. Liu, J. Hewson, J. Chen, H. Pitsch, Effects of strain rate on high-pressure nonpremixed n-heptane autoignition in counterflow, Combustion and Flame 137 (3) (2004) 320-339. doi: 10.1016/j.combustflame.2004.01.011

[40] H. Curran, P. Gaffuri, W. Pitz, C. Westbrook, A comprehensive modeling study of n-heptane oxidation, Combustion and Flame 114 (1-2) (1998) 149-177. doi:10.1016/S0010-2180(97) 00282-4

[41] F. Contino, Combustion in homogeneous charge compression ignition engines : Experimental analysis using ethyl esters and development of a method to include detailed chemistry mechanisms in numerical simulations, Ph.D. thesis, Université Catholique de Louvain (2011).

[42] M. Mehl, W. Pitz, M. Sjberg, J. Dec, Detailed kinetic modeling of low-temperature heat release for prf fuels in an hcci engine, SAE Technical paper 2009-01-1806 (2009) 1-cdoi:10.4271/ 2009-01-1806

[43] M. Mehl, W. Pitz, C. Westbrook, H. Curran, Kinetic modeling of gasoline surrogate components and mixtures under engine conditions, Proc Combustion Inst 33 (1) (2011) 193-200. doi:10.1016/j.proci.2010.05.027

[44] F. Contino, H. Jeanmart, T. Lucchini, G. D'Errico, Coupling of in situ adaptive tabulation and dynamic adaptive chemistry: An effective method for solving combustion in engine simulations, Proc Combustion Ins doi : 10.1016/j .proci.2010.08.002

[45] F. Contino, T. Lucchini, G. D’Errico, C. Duynslaegher, V. Dias, H. Jeanmart, Simulations of advanced combustion modes using detailed chemistry combined with tabulation and mechanism reduction techniques, SAE Technical Paper 2012-01-0145 doi: 10.4271/2012-01-0145

[46] A. Babajimopoulos, D. Assanis, D. Flowers, S. Aceves, R. Hessel, A fully coupled computational fluid dynamics and multizone model with detailed chemical kinetics for the simulation of premixed charge compression ignition engines, Int J Engine Res 6 (5) (2005) 497-512. doi : 10.1243/146808705X30503

[47] M. Raju, M. Wang, M. Dai, W. Piggott, D. Flowers, Acceleration of detailed chemical kinetics using multi-zone modeling for cfd in internal combustion engine simulations, SAE Technical Paper, 2012-01-0135 doi : 10.4271/2012-01-0135

[48] T. D. Cuyper, J. Demuynck, S. Broekaert, M. D. Paepe, S. Verhelst, Heat transfer in premixed spark ignition engines part ii: Systematic analysis of the heat transfer phenomena, Energy 116 


\section{Appendix A. Equilibrium Wall Model Formulation}

In the tables below, you can find an overview of the used equilibrium wall models, with the description of their thermal profile in the boundary layer and the equation for the wall heat flux that follows from that.

\begin{tabular}{|c|c|c|c|}
\hline Model & Temperature profile & Valid $\mathbf{y}^{+}$ & $\mathbf{T}^{+}$ \\
\hline \multirow[t]{2}{*}{ Launder and Spalding [20] } & $T^{+}=\operatorname{Pry}^{+}$ & $\mathrm{y}^{+}<11$ & $T^{+}=\frac{\rho u_{\tau} c_{p}\left(T_{w}-T\right)}{q_{w}}$ \\
\hline & $T^{+}=\operatorname{Pr}_{t}\left(\frac{1}{k} \ln \left(y^{+}\right)+B\right)+P$ & $\mathrm{y}^{+} \geq 11$ & \\
\hline \multirow[t]{2}{*}{ Angelberger et al. [22] } & $T^{+}=\operatorname{Pry}^{+}$ & $\mathrm{y}^{+}<11$ & $T^{+}=\frac{\rho u_{\tau} c_{p} T}{q_{w}} \ln \left(\frac{T_{w}}{T}\right)$ \\
\hline & $T^{+}=P r_{t}\left(\frac{1}{k} \ln \left(y^{+}\right)+B\right)+P$ & $\mathrm{y}^{+} \geq 11$ & \\
\hline Han and Reitz [23] & $T^{+}=2.1 \ln \left(y^{+}\right)+2.5$ & $\mathrm{All} \mathrm{y}^{+}$ & $T^{+}=\frac{\rho u_{\tau} c_{p} T}{q_{w}} \ln \left(\frac{T_{w}}{T}\right)$ \\
\hline \multirow[t]{3}{*}{ Huh et al. [21] } & $T^{+}=\operatorname{Pry}^{+}-0.5 \operatorname{Pr} S^{+}\left(y^{+}\right)^{2}$ & $\mathrm{y}^{+}<11$ & $T^{+}=\frac{\rho u_{\tau} c_{p}\left(T_{w}-T\right)}{q_{w}}$ \\
\hline & $T^{+}=13.2 \operatorname{Pr}+2.195 \ln \left(y^{+}\right)-5.66$ & $\mathrm{y}^{+} \geq 11$ & \\
\hline & $-S^{+}\left(87.12 \operatorname{Pr}+2.198 y^{+}-28.98\right)$ & & \\
\hline Rakopoulos et al. [6] & $\begin{aligned} T^{+}= & \frac{1}{0.4767}\left[\ln \left(y^{+}+\frac{1}{0.4767 P r}\right)-\ln \left(40+\frac{1}{0.4767 P r}\right)\right] \\
& +10.2384+P^{+}\left(\frac{y^{+}-40+117.31\left(0.4767+\frac{1}{P r}\right)}{0.4767+\frac{1}{P r}}\right)\end{aligned}$ & All $y^{+}$ & $T^{+}=\frac{\rho u_{\tau} c_{p} T}{q_{w}} \ln \left(\frac{T_{w}}{T}\right)$ \\
\hline
\end{tabular}

Table A.1: Thermal wall models

\begin{tabular}{|c|c|c|}
\hline Model & Wall heat flux & Valid $\mathbf{y}^{+}$ \\
\hline \multirow[t]{2}{*}{ Launder and Spalding [20] } & $q_{w}=\frac{\rho c_{p} u_{\tau}\left(T_{w}-T\right)}{P r y^{+}}$ & $\mathrm{y}^{+}<11$ \\
\hline & $q_{w}=\frac{\rho c_{p} u_{\tau}\left(T_{w}-T\right)}{\operatorname{Pr}\left(\frac{1}{k} \ln \left(y^{+}\right)+B\right)+P}$ & $\mathrm{y}^{+} \geq 11$ \\
\hline \multirow[t]{2}{*}{ Angelberger et al. [22] } & $q_{w}=\frac{\rho c_{p} u_{r} T \ln \left(T_{w} / T\right)}{P r y^{4}}$ & $\mathrm{y}^{+}<11$ \\
\hline & $q_{w}=\frac{\rho c_{p} u_{t} T \ln \left(T_{w} / T\right)}{\operatorname{Pr}\left(\frac{1}{k} \ln \left(y^{+}\right)+B\right)+P}$ & $\mathrm{y}^{+} \geq 11$ \\
\hline Han and Reitz [23] & $q_{w}=\frac{\rho c_{p} u_{T} T \ln \left(T_{w} / T\right)}{2.1 \ln \left(y^{+}\right)+2.5}$ & ${\text { All } y^{+}}^{+}$ \\
\hline \multirow[t]{2}{*}{ Huh et al. [21] } & $q_{w}=\frac{\rho c_{p} u_{\tau}\left(T_{w}-T\right)-0.5 \operatorname{Pr} \frac{d P}{d t} \frac{v}{u_{\tau}}\left(y^{+}\right)^{2}}{\operatorname{Pry}^{+}}$ & $\mathrm{y}^{+}<11$ \\
\hline & $q_{w}=\frac{\rho c_{p} u_{\tau}\left(T_{w}-T\right)-\frac{d P}{d t} \frac{v}{u_{\tau}}\left(87.12 P r+2.195 y^{+}-28.98\right)}{13.2 P r+2.195 \ln \left(y^{+}\right)-5.66}$ & $\mathrm{y}^{+} \geq 11$ \\
\hline \multirow{2}{*}{ Rakopoulos et al. [6] } & $\left.\rho c_{p} u_{\tau} T \ln \frac{T_{W}}{T}\right)-\frac{d P}{d t} \frac{v}{u_{\tau}}\left(\frac{y^{+}-40}{0.4767+\frac{1}{P T}}+117.31\right)$ & \multirow{2}{*}{ All $\mathrm{y}^{+}$} \\
\hline & $q_{w}-\frac{1}{\frac{1}{0.4767}\left[\ln \left(y^{+}+\frac{1}{0.4767 P_{r}}\right)-\ln \left(40+\frac{1}{0.4767 P_{r}}\right)\right]+10.2384}$ & \\
\hline
\end{tabular}

Table A.2: Wall heat flux formulation for the different models 